\title{
Universiteit
}

Leiden

The Netherlands

\section{Poly(ethylene glycol)-based stable isotope labeling reagents for the quantitative analysis of low molecular weight metabolites by LC-MS} Abello, N.; Geurink, P.P.; Toorn, M. van der; Oosterhout, A.J.M. van; Lugtenburg, J.; Marel, G.A. van der; ... ; Bischoff, R.

\section{Citation}

Abello, N., Geurink, P. P., Toorn, M. van der, Oosterhout, A. J. M. van, Lugtenburg, J., Marel, G. A. van der, ... Bischoff, R. (2008). Poly(ethylene glycol)-based stable isotope labeling reagents for the quantitative analysis of low molecular weight metabolites by LCMS. Analytical Chemistry, 80(23), 9171-9180. doi:10.1021/ac801215c

Version: $\quad$ Publisher's Version

License: $\quad$ Licensed under Article 25fa Copyright Act/Law (Amendment Taverne)

Downloaded from: https://hdl.handle.net/1887/3239443

Note: To cite this publication please use the final published version (if applicable). 


\title{
Poly(ethylene glycol)-Based Stable Isotope Labeling Reagents for the Quantitative Analysis of Low Molecular Weight Metabolites by LC-MS
}

\author{
Nicolas Abello, ${ }^{\dagger}$ Paul P. Geurink, ${ }^{\ddagger}$ Marco van der Toorn, ${ }^{\S}$ Antoon J. M. van Oosterhout, $\$$ \\ Johan Lugtenburg, ${ }^{\ddagger}$ Gijs A. van der Marel," Huib A. M. Kerstjens," Dirkje S. Postma," \\ Hermen S. Overkleeft, ${ }^{*, *}$ and Rainer Bischoff*,t

\begin{abstract}
Department of Analytical Biochemistry, University of Groningen, Groningen, The Netherlands, Department of Bio-organic Synthesis, Leiden University, Leiden, The Netherlands, and Laboratory of Allergology and Pulmonary Diseases, Department of Pulmonary Diseases, University Medical Center Groningen, University of Groningen, Groningen, The Netherlands
\end{abstract}

Stable isotope labeling (SIL) in combination with liquid chromatography-mass spectrometry is one of the most widely used quantitative analytical methods due to its sensitivity and ability to deal with extremely complex biological samples. However, SIL methods for metabolite analysis are still often limited in terms of multiplexing, the chromatographic properties of the derivatized analytes, or their ionization efficiency. Here we describe a new family of reagents for the SIL of primary amine-containing compounds based on pentafluorophenyl-activated esters of ${ }^{13} \mathrm{C}$-containing poly(ethylene glycol) chains (PEG) that addresses these shortcomings. A sequential buildup of the PEG chain allowed the introduction of various numbers of ${ }^{13} \mathrm{C}$ atoms opening extended multiplexing possibilities. The PEG derivatives of rather hydrophilic molecules such as amino acids and glutathione were successfully retained on a standard $C_{18}$ reversed-phase column, and their identification was facilitated based on $\mathrm{m} / \mathrm{z}$ values and retention times using extracted ion chromatograms. The mass increase due to PEG derivatization moved low molecular weight metabolite signals out of the often noisy, low $\mathrm{m} / \mathrm{z}$ region of the mass spectra, which resulted in enhanced overall sensitivity and selectivity. Furthermore, elution at increased retention times resulted in efficient electrospray ionization due to the higher acetonitrile content in the mobile phase. The method was successfully applied to the quantification of intracellular amino acids and glutathione in a cellular model of human lung epithelium exposed to cigarette smoke-induced oxidative stress. It was shown that the concentration of most amino acids increased upon exposure of A549 cells to gas-phase cigarette smoke with respect to air control

\footnotetext{
* To whom correspondence should be addressed. Concerning analytical chemistry. E-mail: r.p.h.bischoff@rug.nl. Tel.: +3150363 3338. Fax: +3150363 7582. Concerning synthetic chemistry. E-mail: h.s.overkleeft@chem.leidenuniv.nl. Tel.: +31 71527 4342. Fax: +31 715274307 .

$\dagger$ Department of Analytical Biochemistry, University of Groningen.

* Department of Bio-organic Synthesis, Leiden University.

$\S$ Laboratory of Allergology and Pulmonary Diseases, University Medical Center Groningen, University of Groningen.

"Department of Pulmonary Diseases, University Medical Center Groningen, University of Groningen.
}

and cigarette smoke extract and that free thiol-containing species (e.g., glutathione) decreased although disulfide bond formation was not increased. These labeling reagents should also prove useful for the labeling of peptides and other compounds containing primary amine functionalities.

The use of mass spectrometry as a quantitative analytical technique is hampered by the fact that different compounds do not ionize with the same efficiency. Furthermore, matrix effects summarized under the term "ionization suppression" may occur when several compounds coelute and thus "compete" for ionization. Finally, slight variations of parameter settings of the instrument and slightly changing chromatographic conditions between different liquid chromatography-mass spectrometry (LC-MS) runs can affect the magnitude of the resulting signals. To overcome these limitations, quantification based on stable isotope labeling (SIL) has found widespread use. SIL allows the absolute quantification of a known compound by comparing it with a stable isotope-containing standard added to the sample at a known concentration or the relative quantification of many compounds in several samples labeled with reagents containing different numbers of stable isotopes (multiplexing). SIL in combination with LC-MS has therefore become one of the most widely used quantitative analytical methods due to its sensitivity and ability to quantify compounds in extremely complex biological samples.

While stable isotopes can be incorporated into molecules biosynthetically (metabolic labeling), ${ }^{1}$ most compounds require chemical synthesis or chemical derivatization for the introduction of stable isotopes with the exception of ${ }^{18} \mathrm{O},{ }^{2}$ which can be introduced into peptides enzymatically. Chemical derivatization with stable isotope-containing reagents requires the presence of certain functional groups, notably thiols or primary amines.

The growing area of metabolomics requires the quantification of many analytes in highly complex samples. Multiplexing would eventually allow the performance of metabolomics experiments on a larger scale and with greater throughput.

(1) Oda, Y.; Huang, K.; Cross, F. R.; Cowburn, D.; Chait, B. T. Proc. Natl. Acad. Sci. U. S. A. 1999, 96, 6591-6596.

(2) Yao, X.; Freas, A.; Ramirez, J.; Demirev, P. A.; Fenselau, C. Anal. Chem. 2001, 73, 2836-2842.

Analytical Chemistry, Vol. 80, No. 23, December 1, 2008 9171 
However, to date there are few generally applicable stable isotope labeling methods available that allow the LC-MS analysis of a wide range of metabolites and other low molecular weight, hydrophilic compounds (e.g., amino acids) offering the option of extensive multiplexing. Numerous methods have been developed during the past 50 years for the analysis of amino acids by reversed-phase liquid chromatography after derivatization with light-absorbing or fluorescent groups (for an overview, see the introduction of Yang et al. $\left.{ }^{3}\right)$. More recently, methods were developed with the goal of using LC-MS. Yang et $\mathrm{al}^{3}{ }^{3}$ developed a SIL method based on $\mathrm{N}$-alkylnicotinic acid $\mathrm{N}$-hydroxysuccinimide esters (NHS) for the quantification of amino acids by LC-MS. This method allows efficient and relatively fast separation of amino acids on a $\mathrm{C}_{18}$ reversed-phase column with improved ionization efficiency. The isotopologues of the labeling reagents contain either nine ${ }^{1} \mathrm{H}$ or nine ${ }^{2} \mathrm{H}$ atoms, which allow duplex analyses. Due to derivatization with deuterium-labeled reagents, a slight but noticeable effect on retention time was observed, in agreement with earlier reports that had described an effect of deuterium labeling on retention time. ${ }^{4,5}$ Guo et al. ${ }^{4}$ described an alternative method for SIL resulting in dimethylation of primary amines through reductive amination with formaldehyde in its hydrogen- or deuteriumcontaining form allowing duplex analyses of amino acids and amines in urine. Although this method has the advantage of being rather inexpensive, it requires the use of sodium cyanoborohydride, a rather toxic chemical, and does not allow extensive multiplexing. Furthermore, hydrophobicity of the derivatized molecules is only slightly increased, which resulted in a number of the most hydrophilic amino acids eluting in the void volume of a standard $\mathrm{C}_{18}$ reversed-phase column. As a consequence, it was necessary to analyze these amino acids separately by hydrophilic interaction liquid chromatography (HILIC)-MS. Methyl acetimidate-based ${ }^{13} \mathrm{C}$-SIL was used by Shortreed et al. for amino acid analysis. ${ }^{6}$ However, the increase in hydrophobicity was limited and amino acids had to be separated by HILIC instead of the more traditional reversedphase LC. Moreover, with only two carbon atoms, no oxygen and one nitrogen, possibilities of multiplexing using this labeling reagent are rather limited.

In the present article, we report the synthesis and application of pentafluorophenyl-activated esters of poly(ethylene glycol) derivatives (PEG-OPFP) for SIL of primary amine-containing molecules. This family of reagents is based on the amine-specific PFP ester and a mildly hydrophobic PEG chain containing increasing numbers of ${ }^{13} \mathrm{C}$ atoms (Figure 1). The PEG moiety added to the target molecules was designed to do the following: (i) allow stable isotope labeling in a modular fashion without affecting the chromatographic retention time, thus permitting multiplexing beyond presently available strategies, (ii) render low molecular weight metabolites, such as amino acids, sufficiently hydrophobic to be retained on $\mathrm{C}_{18}$ reversed-phase columns, (iii) produce derivatives with comparable or improved MS responses

(3) Yang, W. C.; Mirzaei, H.; Liu, X.; Regnier, F. E. Anal. Chem. 2006, 78, 4702-4708.

(4) Guo, K.; Ji, C.; Li, L. Anal. Chem. 2007, 79, 8631-8638.

(5) Zhang, R.; Sioma, C. S.; Wang, S.; Regnier, F. E. Anal. Chem. 2001, 73, 5142-5149.

(6) Shortreed, M. R.; Lamos, S. M.; Frey, B. L.; Phillips, M. F.; Patel, M.; Belshaw, P. J.; Smith, L. M. Anal. Chem. 2006, 78, 6398-6403.

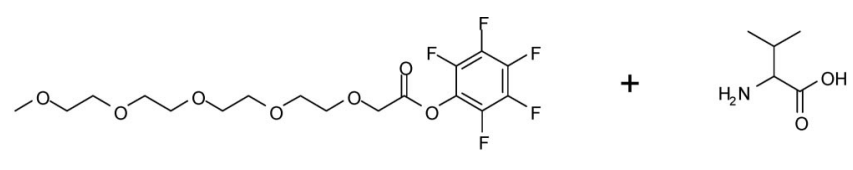

PEG-OPFP

Val
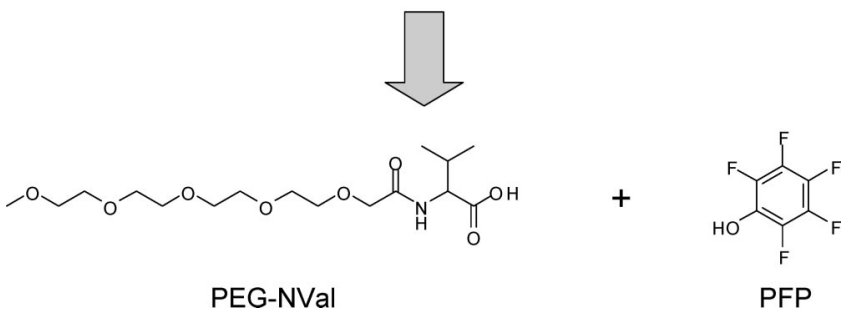

Figure 1. PEG-OPFP-based labeling exemplified with valine. The pentaflurophenyl poly(ethylene glycol) ester (PEG-OPFP) is constituted of 5 ethylene glycol units functionalized with a pentafluorophenyl ester. The latter reacts with the primary amine of an amino acid (in this case, valine) to form a N-derivatized amino acid (PEG-NVal) and PFP as byproduct.

with respect to underivatized compounds upon electrospray ionization (ESI), and (iv) increase the mass of low molecular weight metabolites to move them out of the often noisy, low $\mathrm{m} / \mathrm{z}$ region of mass spectra. All of these properties greatly facilitate quantification of these compounds by LC-MS.

The approach has been applied to the quantification of intracellular amino acids and glutathione in a cellular model of human lung epithelium exposed to cigarette smoke-induced oxidative stress. Results show that amino acids can be quantified in absolute terms and complex samples can be compared relative to each other based on labeling with reagents containing different numbers of ${ }^{13} \mathrm{C}$ atoms. This application shows that SIL based on ${ }^{13} \mathrm{C}$-PEG-OPFP derivatives is a promising method for quantification of low molecular weight, hydrophilic compounds. Initial data show that PEG-OPFPs are also useful for the labeling of peptides.

\section{MATERIALS AND METHODS}

Synthesis of PEG-OPFP Labeling Reagent. Synthesis details are presented as part S-1 of Supporting Information.

General Procedure. Tetrahydrofuran (THF) was distilled over $\mathrm{LiAlH}_{4}$ before use. Methanol $(\mathrm{MeOH})$, diethyl ether $\left(\mathrm{Et}_{2} \mathrm{O}\right)$, dichloromethane (DCM) were of AR grade, purchased at Biosolve (Valkenswaard, The Netherlands), and used as received. All general chemicals (Fluka, Acros, TCI, Aldrich, Sigma) were used as received. $\left[1-{ }^{13} \mathrm{C}_{1}\right]$ Acetic acid and $\left[1,2^{13} \mathrm{C}_{2}\right]$ acetic acid were from Buchem B.V (Apeldoorn, The Netherlands). $\left[1-{ }^{13} \mathrm{C}_{1}\right]$ Bromoacetic acid and $\left[1,2-{ }^{13} \mathrm{C}_{2}\right]$ bromoacetic acid were prepared from $\left[1-{ }^{13} \mathrm{C}_{1}\right]$ acetic acid and $\left[1,2{ }^{13} \mathrm{C}_{2}\right]$ acetic acid via a reported procedure. $^{7}$ Solvents that were used in reactions were stored over $4-\AA$ molecular sieves, except for methanol, which was stored over $3-\AA \AA \AA$ molecular sieves. Molecular sieves were flame dried before use. Amberlite IR120 H resin (Acros, Geel, Belgium)) was rinsed with $\mathrm{MeOH}(4 \times)$ and $\mathrm{Et}_{2} \mathrm{O}(4 \times)$ before use. Column chromatography was performed on silica gel (Screening Devices B.V., Amersfoort, The Netherlands) with a particle size of $40-63 \mu \mathrm{m}$ and a pore size of $60 \AA$ A . Automated column chromatography was performed on a CombiFlash Companion with a Silicycle FLH-R10030B-ISO12

(7) Ouwerkerk, N.; van Boom, J. H.; Lugtenburg, J.; Raap, J. Eur. J. Org. Chem. 2000, 2000, 861-866. 


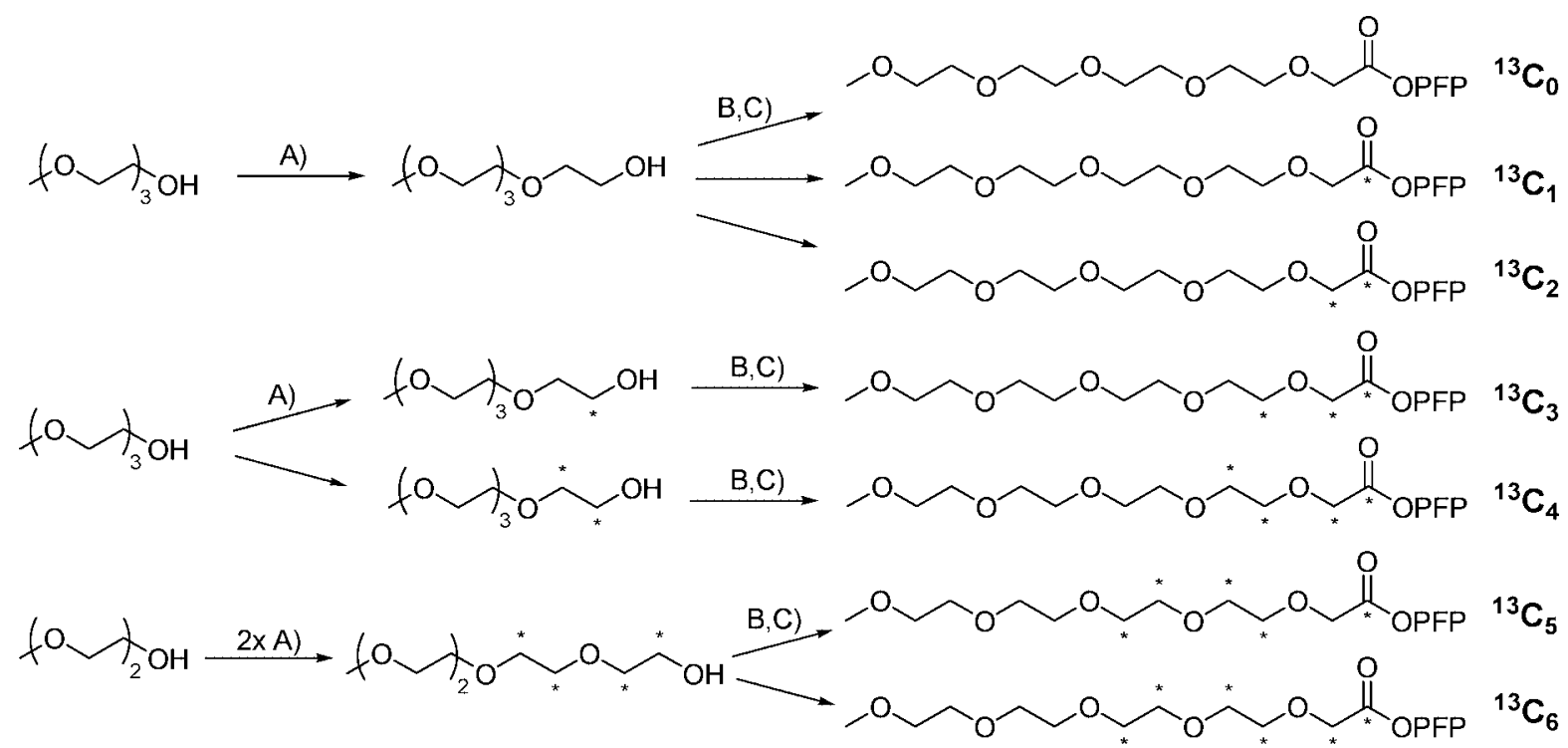

${ }^{a}$ The asterisks (*) indicate the positions of carbon-13 atoms. Reagents and conditions: (A) (i) ${ }^{12} \mathrm{C}_{2}, 1-{ }^{13} \mathrm{C}_{1}$, or $1,2-{ }^{13} \mathrm{C}_{2}-\mathrm{BrAcOH}(1.2$ equiv), $\mathrm{NaH}$ (4 equiv), Nal (catalytic), THF; (ii) $\mathrm{LiAlH}_{4}$ (2.5 equiv) $15 \mathrm{~min}$, then $2 \mathrm{M} \mathrm{NaOH}$ (aq), yield: $75-95 \%$. (B) (i) $\mathrm{BrAcOH}$ (1.2 equiv), $\mathrm{NaH}$ (4 equiv), Nal (catalytic) THF; (ii) Amberlite $\mathrm{H}^{+}$. (C) PFPOH (2 equiv), EDCl (2 equiv), DCM, yield: $55-60 \%$ over two steps.

Sillaflash 12-g cartridge. Eluents ethyl acetate and petroleum ether (40-60) were distilled prior to use. TLC analysis was conducted on Merck aluminum sheets (Silica gel $60 \mathrm{~F}_{254}$, Merck KGaA, Darmstadt, Germany). Compounds were visualized by UV absorption $(254 \mathrm{~nm})$ or by spraying with a solution of $\mathrm{KMnO}_{4}(20 \mathrm{~g} / \mathrm{L})$ and $\mathrm{K}_{2} \mathrm{CO}_{3}(10 \mathrm{~g} / \mathrm{L})$ in water followed by charring at $\pm 150{ }^{\circ} \mathrm{C} .{ }^{1} \mathrm{H}$ and ${ }^{13} \mathrm{C}$ NMR spectra were recorded on a Jeol JNM-FX-200 (200 MHz) (Welwyn Garden City, UK) or a Bruker DMX-600 (600 $\mathrm{MHz}$ ) spectrometer (Bruker BioSpin, Wormer, The Netherlands). Chemical shifts are given in $\mathrm{ppm}(\delta)$ relative to tetramethylsilane ( $\left.{ }^{1} \mathrm{H} \mathrm{NMR}\right)$ or $\mathrm{CDCl}_{3}\left({ }^{13} \mathrm{C} \mathrm{NMR}\right)$ as internal standard. LC-MS analysis was performed on a Finnigan Surveyor HPLC system with a Gemini $\mathrm{C}_{18} 50 \times 4.6 \mathrm{~mm}$ column (Phenomenex, Torrance, $\mathrm{CA}$ ) (detection at 200-600 nm), coupled to a Thermo Finnigan LCQ Advantage Max mass spectrometer (Breda, The Netherlands) with ESI (system 1) and a Jasco HPLC system (Jasco Benelux B.V., IJsselstein, The Netherlands) with a Gemini $3-\mu \mathrm{m} \mathrm{C}_{18} 50 \times 4.6$ $\mathrm{mm}$ column (detection simultaneously at 214 and $254 \mathrm{~nm}$ ), coupled to a PE Sciex API 165 mass spectrometer (Nieuwekerk a/d IJssel, The Netherlands) with ESI (system 2). HPLC gradients were $10-90$ or $0-20 \%$ acetonitrile in $0.1 \%$ TFA-water. The flow rate was $1 \mathrm{~mL} / \mathrm{min}$. High-resolution mass spectra were recorded by direct injection ( $2 \mu \mathrm{L}$ of a $2 \mu \mathrm{M}$ solution in water/acetonitrile; $50 / 50 ; \mathrm{v} / \mathrm{v}$ and $0.1 \%$ formic acid) on a Thermo Finnigan LTQ Orbitrap mass spectrometer equipped with an ESI ion source in positive mode (source voltage $3.5 \mathrm{kV}$, sheath gas flow 10 , capillary temperature $250{ }^{\circ} \mathrm{C}$ ) with resolution $R=60000$ at $m / z 400$ (mass range $m / z=150-2000)$ and dioctyl phthalate $(m / z=391.284$ 28) as a "lock mass". ${ }^{8}$ The high-resolution mass spectrometer was calibrated prior to measurements with a calibration mixture (Thermo Finnigan).

General Procedure A: Extension of the PEG Chain by One Ethylene Glycol Unit (see Scheme 1). NaH (60\% in mineral oil, 4

(8) Olsen, J. V.; de Godoy, L. M.; Li, G.; Macek, B.; Mortensen, P.; Pesch, R.; Makarov, A.; Lange, O.; Horning, S.; Mann, M. Mol. Cell. Proteomics 2005, 4, 2010-2021. equiv) was carefully added in portions to a solution of the alcohol (1 equiv), bromoacetic acid (either ${ }^{12} \mathrm{C}_{2}, 1-{ }^{13} \mathrm{C}_{1}$, or $1,2-{ }^{13} \mathrm{C}_{2}, 1.2$ equiv), and $\mathrm{NaI}$ ( 0.01 equiv) in THF $(5 \mathrm{~mL} / \mathrm{mmol})$. After complete conversion of the alcohol (monitored by TLC analysis, usually after $2 \mathrm{~h}$ ), $\mathrm{LiAlH}_{4}$ (2.5 equiv) was carefully added in portions. The reaction was stirred for 30 min after which $\mathrm{NaOH}$ (as a $2 \mathrm{M}$ aqueous solution, 2.5 equiv) was added dropwise. After $15 \mathrm{~min}$ of stirring, $\mathrm{Et}_{2} \mathrm{O}(5 \mathrm{~mL} / \mathrm{mmol})$ and $\mathrm{MgSO}_{4}$ were added. The reaction was stirred vigorously for another $5 \mathrm{~min}$ after which all solids were filtered off over Celite. The filtrate was concentrated under reduced pressure and the resulting crude product was purified by column chromatography on silica gel (100\% EtOAc $\rightarrow 15 \%$ $\mathrm{MeOH} / \mathrm{EtOAc})$.

General Procedure B: Reaction of a PEG Chain Alcohol with Bromoacetic Acid Followed by Acidic Workup (See Scheme 1). NaH (60\% in mineral oil, 4 equiv) was carefully added in portions to a solution of the alcohol (1 equiv), bromoacetic acid (either ${ }^{12} \mathrm{C}_{2}$, $1-{ }^{13} \mathrm{C}_{1}$, or $1,2-{ }^{13} \mathrm{C}_{2}, 1.2$ equiv), and $\mathrm{NaI}$ ( 0.01 equiv) in THF (5 mL/ $\mathrm{mmol}$ ). After complete conversion of the alcohol (monitored by TLC analysis, usually after $2 \mathrm{~h}$ ), Amberlyte IR120 $\mathrm{H}$ resin and $\mathrm{Et}_{2} \mathrm{O}(2 \mathrm{~mL} / \mathrm{mmol})$ were added. After $30 \mathrm{~min}$ of stirring, all solids and the resin were filtered off over Celite and the filtrate was concentrated under reduced pressure. The crude product was subjected to the next step (general procedure C) without further purification.

General Procedure C: Conversion of the Carboxylic Acid to the Pentafluorophenyl Ester (See Scheme 1). The crude carboxylic acid (obtained in general procedure B, 1 equiv) was dissolved in DCM $(5 \mathrm{~mL} / \mathrm{mmol})$. To this solution were added pentafluorophenol (2 equiv) and 1-ethyl-3-(3'-dimethylaminopropyl)carbodiimide (EDCI) ( 2 equiv), and the mixture was stirred for $2 \mathrm{~h}$. The latter was then concentrated under reduced pressure, and the product was purified by automated column chromatography on silica gel (10\% $\rightarrow 80 \% \mathrm{EtOAc} /$ petroleum ether). 
Labeling of a Standard Solution of Amino Acids and Glutathione. ${ }^{13} \mathrm{C}$-Containing PEG-OPFP reagents were diluted to $100 \mathrm{mM}$ in dimethyl sulfoxide (DMSO; Merck KGaA). A solution of all 20 natural amino acids (Sigma; Zwijndrecht, The Netherlands) and glutathione (Merck KGaA) at various concentrations $(0,0.1,0.5,1,10,30,50 \mu \mathrm{M}$ each) in $100 \mathrm{mM}$ sodium phosphate buffer, $\mathrm{pH} 8$, was labeled with $5 \mathrm{mM}$ PEG-OPFP for $10 \mathrm{~min}$ in a thermomixer (Eppendorf; Hamburg, Germany) at 450 $\mathrm{rpm}, 25^{\circ} \mathrm{C}$. Esterification of tyrosine was reversed by incubating the sample for $15 \mathrm{~min}$ in a boiling water bath as previously described for $N$-hydroxysuccinimide ester-based acylation. ${ }^{9} \mathrm{Di}$ sulfide bonds and residual thioesters were cleaved by an extra 5 -min incubation in a boiling water bath in the presence of 80 $\mathrm{mM}$ dithiothreitol (DTT; Sigma). Finally, formic acid (Merck $\mathrm{KGaA}$ ) was added to the samples prior to $\mathrm{LC}-\mathrm{MS}$ analysis to a final concentration of $1 \%(\mathrm{v} / \mathrm{v})$.

Labeling Efficiency as a Function of pH. ${ }^{13} \mathrm{C}$-Containing PEG-OPFP reagents were diluted to $100 \mathrm{mM}$ in DMSO. A $50 \mu \mathrm{M}$ solution of all 20 natural amino acids and glutathione was labeled as described above in different buffers (100 mM concentration): (i) sodium phosphate, $\mathrm{pH} 7.0$, (ii) sodium phosphate, $\mathrm{pH} 7.5$, (iii) sodium phosphate, $\mathrm{pH} 8.0$, (iv) boric acid, $\mathrm{pH} 8.5$, and (v) boric acid, $\mathrm{pH}$ 9.0.

Labeling of Amino Acids and Glutathione in Cultured A549 Cells Undergoing Cigarette Smoke-Induced Oxidative Stress. The human alveolar type II epithelium-like adherent cell line A549 (American Type Culture Collection, Manassas, VA) was grown in RPMI 1640 medium with $25 \mathrm{mM}$ HEPES, L-glutamine (BioWitthaker; Verviers, Belgium) supplemented with $10 \%$ heatinactivated fetal calf serum (BioWitthaker), and $20 \mu \mathrm{g} / \mathrm{mL}$ gentamicin (Centafarm Services; Etten-Leur, The Netherlands). Cells were grown in 25- $\mathrm{cm}^{2}$ plastic culture flasks (Costar; Cambridge, MA) at $37{ }^{\circ} \mathrm{C}$ in an atmosphere of $5 \% \mathrm{CO}_{2}$ until $80-90 \%$ confluency was reached. Before the experiments, A549 cells were grown for $16 \mathrm{~h}$ in serum-free RPMI 1640 medium.

A549 cells were subsequently exposed to gas-phase cigarette smoke (GPCS; 3 biological replicates), air (3 biological replicates), or a cigarette smoke extract (CSE; 2 biological replicates). For GPCS exposure, the medium was extemporaneously removed and the culture flask was positioned upside down, allowing direct contact of smoke with epithelial cells. Kentucky 2R4F researchreference cigarettes (The Tobacco Research Institute, University of Kentucky; Lexington, KY) were smoked using a peristaltic pump. Cigarette filters were cut and each cigarette was smoked for exactly $5 \mathrm{~min}$ at a rate of $8 \mathrm{~L} / \mathrm{h}$. The smoke was blown through a small plastic tube for direct distribution of GPCS inside the culture flasks. Air was used as negative control under the same conditions. For CSE experiments, cigarettes were prepared and smoked as for GPCS exposure. Four cigarettes were bubbled through $50 \mathrm{~mL}$ of cell culture medium, and this solution was regarded as $100 \%$ strength CSE. Cells were incubated with 10\% CSE at room temperature for $4 \mathrm{~h}$ and subsequently detached with cell dissociation buffer, enzyme-free, PBS-based (Invitrogen; Breda, The Netherlands). This was followed by three wash cycles (addition of $10 \mathrm{~mL}$ of PBS followed by centrifugation at $560 \mathrm{~g}$ for 5 min, at room temperature, and removal of the excess PBS).

(9) Abello, N.; Kerstjens, H. A.; Postma, D. S.; Bischoff, R. J. Proteome Res. 2007, 6, 4770-4776.
Washed cells were resuspended in $1 \mathrm{~mL}$ of $100 \mathrm{mM}$ sodium phosphate $\mathrm{pH} 8,1 \mathrm{mM}$ ethylenediaminetetraacetic acid disodium dihydrate (EDTA; Duchefa Biochemie B.V.; Haarlem, The Netherlands), lysed by sonication, and immediately supplemented with $1 \mathrm{mM}$ phenylmethanesulfonyl fluoride (Sigma). Insoluble material was removed by centrifugation at $20000 \mathrm{~g}$ for $45 \mathrm{~min}$, at $4{ }^{\circ} \mathrm{C}$, and the supernatant was filtered through $5-\mathrm{kDa}$ cutoff centrifugal ultrafiltration units (Ultrafree-0.5, PBCC Biomax Membrane; Millipore; Amsterdam, The Netherlands). After an optional thiol alkylation step with iodoacetamide, amino acids and glutathione in the filtrate were labeled with PEG-OPFP reagents and analyzed by LC-MS. The retentate was recovered, diluted to the initial sample volume with $100 \mathrm{mM}$ sodium phosphate buffer, $\mathrm{pH} \mathrm{8,1}$ mM EDTA, and the protein concentration determined using a Micro BCA Protein Assay Kit (Pierce; Rockford, IL).

Thiol-containing species (i.e., glutathione and cysteine) can be found as reduced free thiols or as disulfides. These forms were discriminated either by alkylating the free thiols with iodoacetamide or by quantifying them globally to give the total amount of thiol-containing compounds. The $180 \mu \mathrm{L}$ of filtrate were supplemented with $10 \mu \mathrm{L}$ of iodoacetamide (Sigma; final concentration, $25 \mathrm{mM}$ ) or buffer (100 mM sodium phosphate buffer, $\mathrm{pH} 8)$. After a 30-min incubation in the dark in a thermomixer at $450 \mathrm{rpm}, 25$ ${ }^{\circ} \mathrm{C}$, the samples were labeled with PEG-OPFP reagents and treated further as described above for the standard solution of amino acids (including a final reduction with DTT). The PEG-OPFP labels contained 1,4 , or $6{ }^{13} \mathrm{C}$ atoms for the samples exposed to air, GPCS, or CSE, respectively. Subsequently, the samples were divided into three groups according to the type of exposure. Twenty microliters of each sample were randomly chosen from each of the three groups and mixed together (since CSE exposure was done only in duplicate, one sample was used twice). The three mixtures (replicates), containing each one GPCS-, one CSE-, and one air-exposed sample were analyzed by LC-MS.

LC-MS. The HPLC part of the analytical system consisted of an Agilent series 1100 capillary LC system (Waldbronn, Germany) comprising a degasser, a binary pump with stream splitter and flow controller, a thermostated autosampler $\left(4^{\circ} \mathrm{C}\right)$, and a thermostated column compartment $\left(40^{\circ} \mathrm{C}\right)$. Labeled amino acids and glutathione were chromatographically separated with an Atlantis $\mathrm{dC} 18$ column (Waters; Etten-Leur, The Netherlands; $1.0 \mathrm{~mm} \times$ $150 \mathrm{~mm}$, particle size $3 \mu \mathrm{m}$ ). Mobile phase A consisted of $0.1 \%$ $(\mathrm{v} / \mathrm{v})$ formic acid in ultrapure water. Mobile phase B was $0.1 \%$ (v/v) formic acid in acetonitrile (HPLC-S gradient grade; Biosolve; Valkenswaard, The Netherlands). Injection volumes were $1 \mu \mathrm{L}$ for calibration curves, $3 \mu \mathrm{L}$ in the case of A549 cell lysates, and $0.5 \mu \mathrm{L}(25 \mathrm{pmol})$ for all other experiments with standard solutions. The separation was performed with an increasing gradient of $\mathrm{B}$ $\left(2-37 \%\right.$ at $\left.0.5 \% \times \min ^{-1}\right)$ at $50 \mu \mathrm{L} \times \min ^{-1}$ flow rate.

The analytes were detected by an Agilent SL ion trap mass spectrometer equipped with an ESI source operated in positive mode. MS data were acquired over a scan range of $200-1200 \mathrm{~m} / \mathrm{z}$ in MS mode. All extracted ion chromatograms (EICs) were retrieved with a tolerance of $\pm 0.5 \mathrm{Da}$. The chromatograms were acquired without rolling average and underwent subsequently Gaussian smoothing (3-point width).

Data Analysis. Integration of all peaks was automated using QuantAnalysis 1.8/Bruker Daltonics DataAnalysis 3.4 (Bruker 
Daltonik GmbH; Bremen, Germany). Peaks were defined by their $m / z$ values $\pm 0.5 \mathrm{Da}$ based on singly charged states and their retention times $\pm 0.5 \mathrm{~min}$ after a 3-point Gaussian smoothing of the EICs. Peak integration was checked manually from time to time.

Peak areas for each amino acid were converted in molar concentrations using calibration curve equations (see Figures S-2-S-22 Supporting Information) except for thiol-containing species, because we did not acquire a calibration curve for the carbamidomethyl derivatives of cysteine and glutathione. Their responses were expressed in ratios. Values (molar concentration or MS response) were subsequently normalized with respect to the total protein concentration.

Due to the small number of replicates (triplicate analyses for each condition), a normal distribution of data points could not be ascertained using $Z$-score transformation. Differences in amino acid and glutathione levels between the three conditions (gasphase cigarette smoke, air, and cigarette smoke extract) were therefore assessed using a nonparametric test (Kruskal-Wallis one-way analysis of variance). A $p$-value below 0.05 was considered significant.

\section{RESULTS AND DISCUSSION}

Synthesis of PEG-OPFP Labeling Reagents. The chemical synthesis was based on the stepwise assembly of PEG chains from building blocks containing 0,1 , or $2{ }^{13} \mathrm{C}$ atoms per ethylene glycol unit. This allowed a high degree of multiplexing and extensive flexibility. The family of PEG-OPFP labeling reagents (with a general structure as depicted in Figure 1) consisted of a poly(ethylene glycol) core (five ethylene glycol units) functionalized with a pentafluorophenyl ester at one end and a methoxy group at the other end. Seven compounds were synthesized (see Scheme 1) with a number of ${ }^{13} \mathrm{C}$ atoms ranging from 0 to 6 . The poly (ethylene glycol)s were built up via "chain extensions" using a two-step-one-pot procedure in which the ethylene glycol hydroxyl function was first alkylated with bromoacetic acid followed by reduction of the formed carboxylic acid (as a sodium salt) to an alcohol with $\mathrm{LiAlH}_{4}$. The newly formed alcohol was purified by column chromatography and used in a subsequent "chain extension step". By using either $1{ }^{13} \mathrm{C}$ - or $1,2{ }^{13} \mathrm{C}_{2}$-bromoacetic acid (synthesized from commercially available $1{ }^{13} \mathrm{C}$ - and $1,2^{-13} \mathrm{C}_{2}$-acetic acid via a previously reported procedure), ${ }^{7}$ a varying number of ${ }^{13} \mathrm{C}$ isotopes was incorporated. The formed carboxylate was converted into its protonated form by adding Amberlite $\mathrm{H}^{+}$ after the last alkylation step instead of reducing it with $\mathrm{LiAlH}_{4}$. Finally, it was converted into its corresponding PFP active ester in a reaction with pentafluorophenol and EDCI.

In order to acylate primary amines, we decided to use PFP esters instead of $N$-hydroxysuccinimide esters, since the final reagents were moreeasily purifiedin thisactivatedform by silicagelchromatography. ${ }^{10-13}$ Next to this more practical consideration, some reports have revealed limits to the specificity of NHS esters with respect to

(10) Hoang, V. M.; Conrads, T. P.; Veenstra, T. D.; Blonder, J.; Terunuma, A.; Vogel, J. C.; Fisher, R. J. J Biomol. Tech. 2003, 14, 216-223.

(11) Kvaratskhelia, M.; Miller, J. T.; Budihas, S. R.; Pannell, L. K.; Le Grice, S. F. Proc. Natl. Acad. Sci. U. S. A. 2002, 99, 15988-15993.

(12) Siezen, R. J.; Coppin, C. M.; Benedek, G. B. Biochem. Biophys. Res. Commun. 1985, 133, 239-247.

(13) Yem, A. W.; Zurcher-Neely, H. A.; Richard, K. A.; Staite, N. D.; Heinrikson, R. L.; Deibel, M. R., Jr J. Biol. Chem. 1989, 264, 17691-17697. the O-acylation of tyrosines ${ }^{3,14-17}$ and of other hydroxyl-containing amino acids in certain sequence contexts. ${ }^{9,18,19}$ Furthermore, PFP esters have a better stability to hydrolysis in aqueous solutions and a superior reactivity for aminolysis, compared to NHS esters. ${ }^{20}$

Multiplexing possibilities, i.e., the number of isotopologues that can be obtained, are extensive using our approach, not even considering the additional options offered through the incorporation of ${ }^{18} \mathrm{O}$ atoms in the ethylene glycol unit. This opens the possibility to compare many different samples in a single LC-MS analysis. ${ }^{21}$

Quantification of Amino Acids and Glutathione. Many low molecular weight biomolecules such as endogenous amino acids are generally too small or too hydrophilic to be retained by reversed-phase columns. Moreover, their small mass and highly variable ionization efficiency render detection by LC-ESI-MS difficult, especially in complex mixtures. The PEG-OPFP reagents were designed to label all metabolites containing primary amines augmenting their mass by $\sim 250 \mathrm{Da}$ (depending on the chosen reagent), increasing their hydrophobicity to be retained on $\mathrm{C}_{18}$ reversed-phase columns and equalizing their ESI response. Based on these properties, it is possible to quantify the 20 natural amino acids plus glutathione in a single reversed-phase LC-MS analysis (Figure 2).

The analytes were well resolved and eluted over a rather large retention time range despite introduction of the rather bulky PEG chain. While arginine and asparagine, as well as valine and methionine, were coeluting under the described chromatographic conditions, they were easily discriminated and quantified using EICs (Figure 3). Aspartic acid and glycine coeluted with the peak of the hydrolyzed PEG-OPFP reagent, but again this does not impede quantification when using EICs. As expected, incorporation of different numbers of ${ }^{13} \mathrm{C}$ atoms did not affect retention time in accordance with results for other ${ }^{13} \mathrm{C}$-labeling reagents $\mathrm{s}^{4,5}$ (see the example of glutamic acid labeled with different PEGOPFP reagents in Figure S-1 in Supporting Information). Interestingly preliminary results with PEG labeling of peptides show that derivatization has only a minor effect on retention time, which makes PEG labeling a suitable approach to render a wide range of molecules amenable to standard $\mathrm{C}_{18}$ reversed-phase HPLC.

As mentioned above, our labeling strategy leads to a significant mass increase $\left(+248 \mathrm{Da}\right.$, for the all ${ }^{12} \mathrm{C}$ version of the label) with the result that all amino acids are detected between 324 (singly labeled glycine) and $643 \mathrm{Da}$ (doubly labeled lysine), a mass range that is better adapted to analysis by ESI-MS, where mass spectra are generally noisy below 100-200 Da. In order to investigate the effect on ionization efficiency, we compared the intensity of the signals for labeled and nonlabeled tryptophan, which is by

(14) Blumberg, S.; Vallee, B. L. Biochemistry 1975, 14, 2410-2419.

(15) Holmquist, B.; Blumberg, S.; Vallee, B. L. Biochemistry 1976, 15, 46754680.

(16) Ishikawa, Y.; Yamamoto, Y.; Otsubo, M.; Theg, S. M.; Tamura, N. Biochemistry 2002, 41, 1972-1980.

(17) Regnier, F. E.; Julka, S. Proteomics 2006, 6, 3968-3979.

(18) Miller, B. T.; Kurosky, A. Biochem. Biophys. Res. Commun. 1993, 196, 461-467.

(19) Miller, B. T.; Rogers, M. E.; Smith, J. S.; Kurosky, A. Anal. Biochem. 1994, 219, 240-248.

(20) Kida, S.; Maeda, M.; Hojo, K.; Eto, Y.; Nakagawa, S.; Kawasaki, K. Chem. Pharm. Bull. (Tokyo) 2007, 55, 685-687.

(21) Bantscheff, M.; Schirle, M.; Sweetman, G.; Rick, J.; Kuster, B. Anal. Bioanal. Chem. 2007, 389, 1017-1031. 


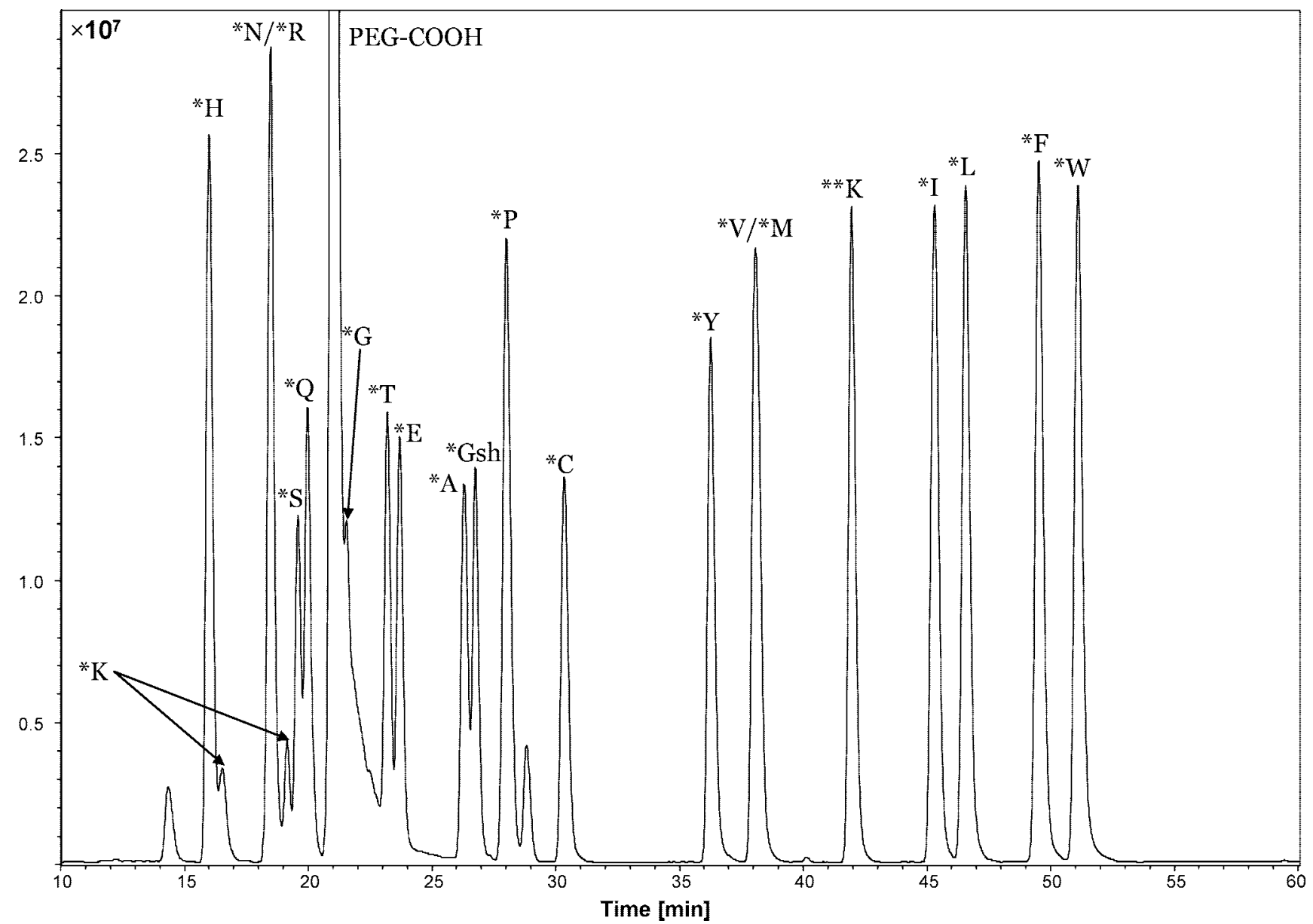

Figure 2. Base peak chromatogram of a standard solution of the 20 natural amino acids plus glutathione (Gsh) (25 pmol injected). Singly and doubly labeled lysine are respectively marked as $\mathrm{K}^{*}$ and $\mathrm{K}^{* *}$. Aspartic acid $(\mathrm{D}, 22.0 \mathrm{~min})$ and glycine $(\mathrm{G}, 22.1 \mathrm{~min})$ coelute with the hydrolyzed excess of reagent (noted PEG-COOH) and are therefore not visible in the base peak chromatogram. They can, however, be quantified in extracted ion chromatograms (see Figure 3).

itself hydrophobic enough to be retained on the reversed-phase column. Signal intensity was 17 -fold higher after derivatization, indicating the beneficial effect of PEG labeling on the ESI response (data not shown). Signal enhancement may in part be due to the fact that tryptophan is more strongly retained after PEG derivatization so that it elutes at a higher percentage of acetonitrile (from 14.3 to $52.0 \mathrm{~min}$ ), where the ESI response is better probably due to more efficient analyte desolvation and improved spray stability. ${ }^{22}$ Since a positive correlation between ESI response and retention time in reversed-phase $\mathrm{LC}$ has been previously demonstrated to be independent of the acetonitrile concentration, ${ }^{23}$ it is likely that all derivatized amino acids exhibit a better ESI response independently of the percentage of ACN at which they elute. Derivatization with PEG may also affect the physicochemical properties of the amino acids favorably with respect to ESI due to its "surfactant-like" properties, resulting in increased surface activity. ${ }^{22}$ Work is in progress to substantiate these findings.

Without incubation in a boiling water bath and the final reduction step with DTT, cysteine is mostly doubly labeled indicating thioester formation (Figure 4, first panel). The 15-min incubation in a boiling water bath, which we normally use to remove nonspecific O-acylation ${ }^{9}$ (in this case, at the tyrosine

(22) Cech, N. B.; Enke, C. G. Mass Spectrom. Rev. 2001, 20, 362-387.

(23) Cech, N. B.; Krone, J. R.; Enke, C. G. Anal. Chem. 2001, 73, 208-213. hydroxyl group) and to hydrolyze the excess of PEG-OPFP, removed most of the thiol labeling, but it also promoted the oxidation of cysteine and glutathione to cystine, glutathione homodimers and glutathione/cysteine (heterodimer) disulfides (Figure 4, second panel). After performing the final reduction step (another 5-min incubation in a boiling water bath in the presence of DTT), neither residual thioesters nor disulfides were observed (Figure 4, third panel) allowing quantification of these analytes.

To allow absolute quantification and to investigate the dynamic range of the LC-MS method, calibration curves were established by injecting $1 \mu \mathrm{L}$ of solutions of all amino acids and of glutathione (labeled with $5 \mathrm{mM} \mathrm{PEG-OPFP)}$ at different concentrations (100 $\mathrm{nM}-50 \mu \mathrm{M}$; Figures S-2-S-22 in Supporting Information). Regression curves fitted to a quadratic model were established over this concentration range with $R^{2}$ values ranging from 0.96995 for glutathione to 0.99994 for isoleucine. Regression curves were linear at low analyte concentrations but often showed a decreasing slope at higher concentrations. This phenomenon is well-known for ESI, where signals level off at high analyte concentrations. ${ }^{22}$ Furthermore, when analytes are present at $50 \mu \mathrm{M}$ each, the molar ratio of PEG-OPFP to primary amines is only 4.5 , without taking side reactions into account. Therefore, labeling may be incomplete at high analyte concentrations (see also further). 

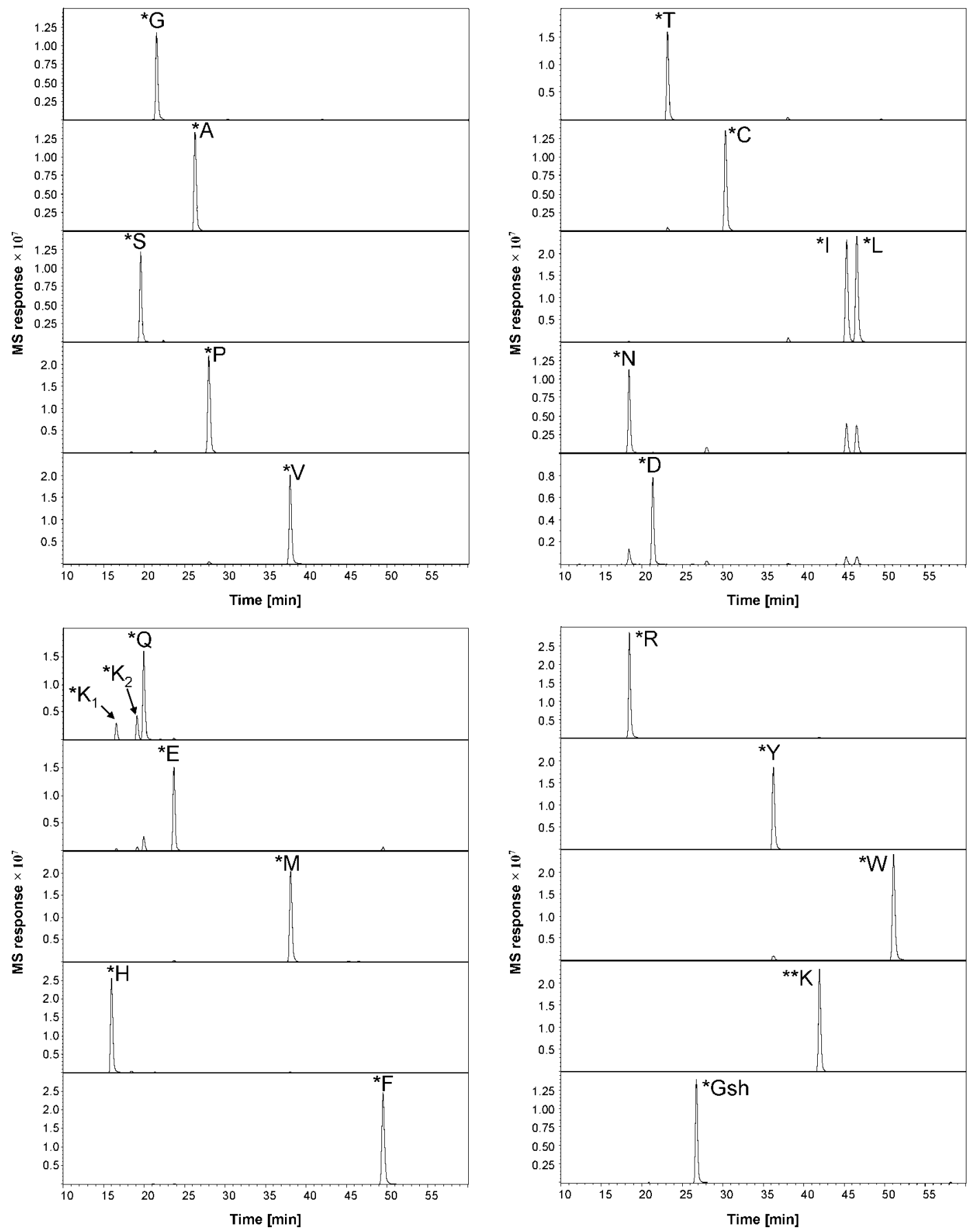

Figure 3. LC-MS analysis of a standard solution of the 20 natural amino acids plus glutathione (Gsh) (25 pmol injected) labeled with PEGOPFP reagent showing extracted ion chromatograms of each derivative. Singly labeled amino acids are marked with an asterisk ( $\left.{ }^{\star} X\right)$ and doubly labeled lysine is marked as ${ }^{* *} \mathrm{~K}$.

pH was shown to influence labeling efficiency, since little singly labeled lysine was observed at $\mathrm{pH} 8.5$ and 9 , whereas the amount increased slightly at $\mathrm{pH} 8$ and further at $\mathrm{pH} 7$ (Figure S-23, panel A, in Supporting Information). The difference in labeling efficiency between $\mathrm{pH} 8.5$ and 8 was, however, minimal for amino acids with a single primary amine and close to optimal at $\mathrm{pH} 8$ (Figure S-23, panel B, in Supporting Information). Since all biological experiments were performed in triplicate at $\mathrm{pH} 8$, we conclude that the reported relative quantifications are reproducible and the observed quantitative changes statistically significant (see Figures 5 and $6)$.
Double derivatization is another possibility to increase derivatization efficiency. We compared simple (5 mM PEG-OPFP) and double derivatization $(2 \times 5 \mathrm{mM}$ PEG-OPFP) of a $50 \mu \mathrm{M}$ standard solution of amino acids and glutathione at pH 8 (Figure S-24 in Supporting Information). Double derivatization resulted in an increase of labeling efficiency to an extent that is comparable to single derivatization at $\mathrm{pH} 8.5$.

Evaluation of Labeling Efficiency. Reproducible and efficient labeling are prerequisites for any stable isotope labeling method. It is thus important to have an easy test to assess labeling efficiency. To this end, we used $100 \mathrm{mM}$ solutions of three 


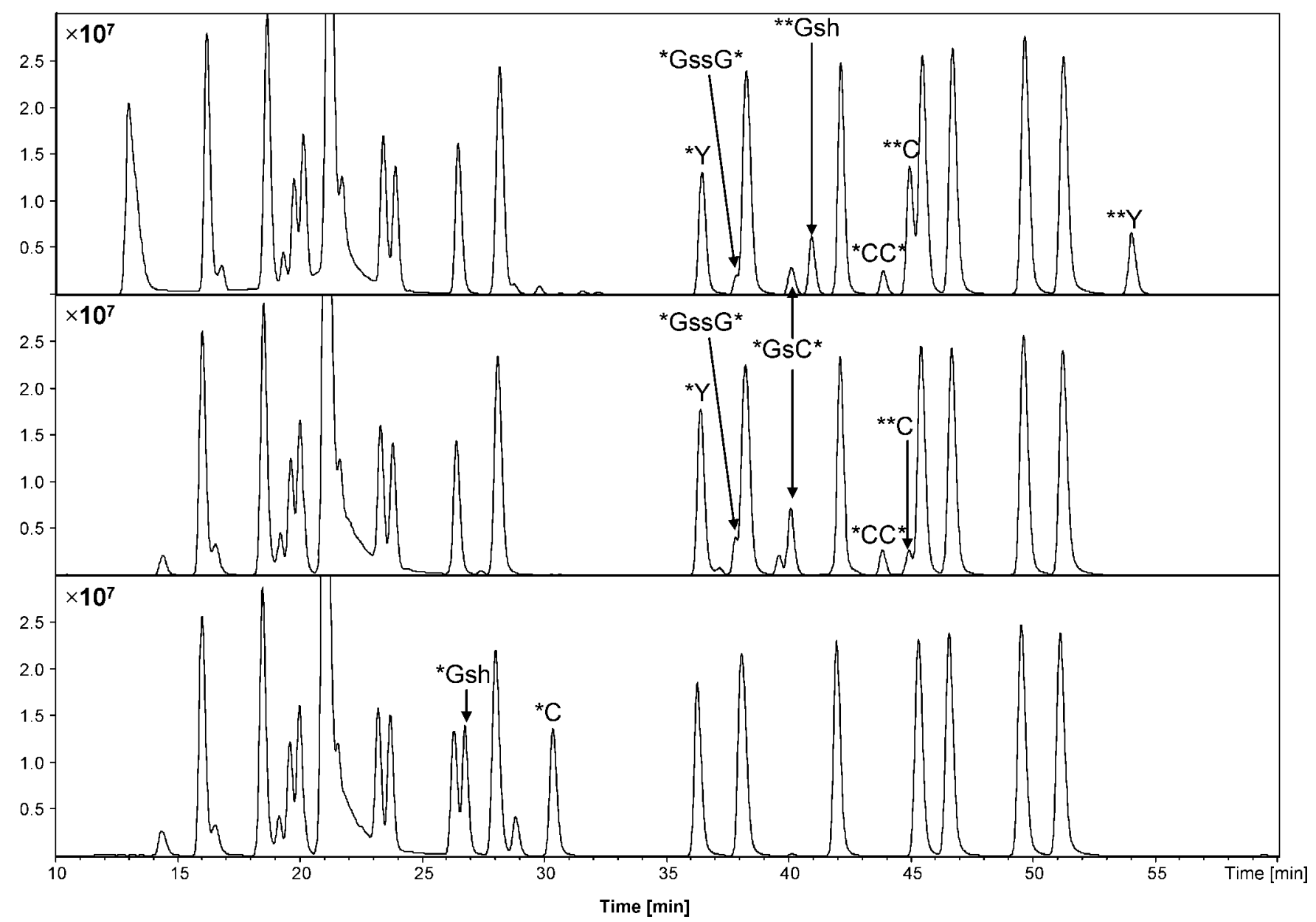

Figure 4. Base peak chromatogram of the standard amino acid mixture (see Figures 2 and 3) after labeling: (first panel) no further treatment, (second panel) with a subsequent 15-min treatment in a boiling water bath, and (third panel) with an additional 5-min treatment with 80 mM DTT. ( ${ }^{*} \mathrm{C}$, singly labeled cysteine; ${ }^{* *} \mathrm{C}$, doubly labeled cysteine; ${ }^{*} \mathrm{CC} *$, doubly labeled cystine, *Gsh, singly labeled glutathione, ${ }^{* *} \mathrm{Gsh}$, doubly labeled glutathione, * $\mathrm{GsC}{ }^{*}$, disulfide glutathione/cysteine, * $\mathrm{Gss} \mathrm{G}^{*}$, disulfide of labeled glutathione, ${ }^{* *} \mathrm{Y}$, doubly labeled tyrosine).

different PEG-OPFP labels (freshly diluted in DMSO) containing respectively 1,4 , or $6{ }^{13} \mathrm{C}$ atoms for the labeling of lysine. This amino acid has two primary amines $(\alpha$ and $\varepsilon$ ) that can react with PEG-OPFP, and efficient labeling should result in a doubly labeled derivative. Investigating labeling efficiency with respect to the production of singly or doubly labeled lysine in standard solutions prior to the cell culture study revealed that labeling efficiency of the reagents containing 1 or $6{ }^{13} \mathrm{C}$ atoms was very similar and close to completeness, whereas the $4{ }^{13} \mathrm{C}$ reagent presented a slightly lower labeling efficiency as shown by higher signals for singly labeled lysines and a signal for doubly labeled lysine representing $\sim 86 \%$ of that of the other labels (Figure S-25 in Supporting Information). While we cannot extrapolate labeling efficiency for all amino acids from this result, we decided to take this difference into account when calculating the relative differences in intracellular amino acid levels in A549 cells, although most of the observed changes were significantly larger than the measured difference of $\sim 15 \%$ for the $4-{ }^{13} \mathrm{C}$ reagent (used for the air controls). We therefore considered a ratio between 0.85 and 1 as not significant even though the Kruskal-Walis test resulted in a significant difference $(\phi<0.05)$.

Quantification of Intracellular Amino Acids and Glutathione in A549 Cells Undergoing Cigarette Smoke-Induced
Oxidative Stress. To investigate whether our labeling method functions in complex biological samples, we initiated a study to determine the intracellular amino acid and glutathione levels in A549 human lung epithelial cells. A549 cells were cultured and exposed to air (control), GPCS, or CSE to mimic conditions in the lung of cigarette smokers. Cell lysates were ultrafiltered at 5 $\mathrm{kDa}$ to separate the protein fraction from the smaller metabolites. The latter were labeled with different PEG-OPFPs containing 1 (GPCS), 4 (air), or 6 (CSE) ${ }^{13} \mathrm{C}$ atoms. The stable isotope-labeled samples were randomly mixed, each mixture containing one of the biological replicates per condition, and analyzed by LC-MS. The peaks corresponding to the 20 natural amino acids and glutathione were integrated in the respective EICs and peak areas converted into molar concentrations based on the calibration curve equations (see Figures S-2-S-22 in Supporting Information). Concentrations were normalized with respect to the total protein concentration as determined in the protein fraction (i.e., the retentate of the $5-\mathrm{kDa}$ ultrafiltration; Table S-1 in Supporting Information). While air- and CSE-treated cells showed similar protein concentrations ( $\sim 675 \mu \mathrm{g} / \mathrm{mL})$, GPCS-treated cells showed clearly reduced protein concentrations $(\sim 275 \mu \mathrm{g} / \mathrm{mL})$. This lower protein concentration may be explained by the fact that GPCStreated cells were more difficult to detach from the culture plate 


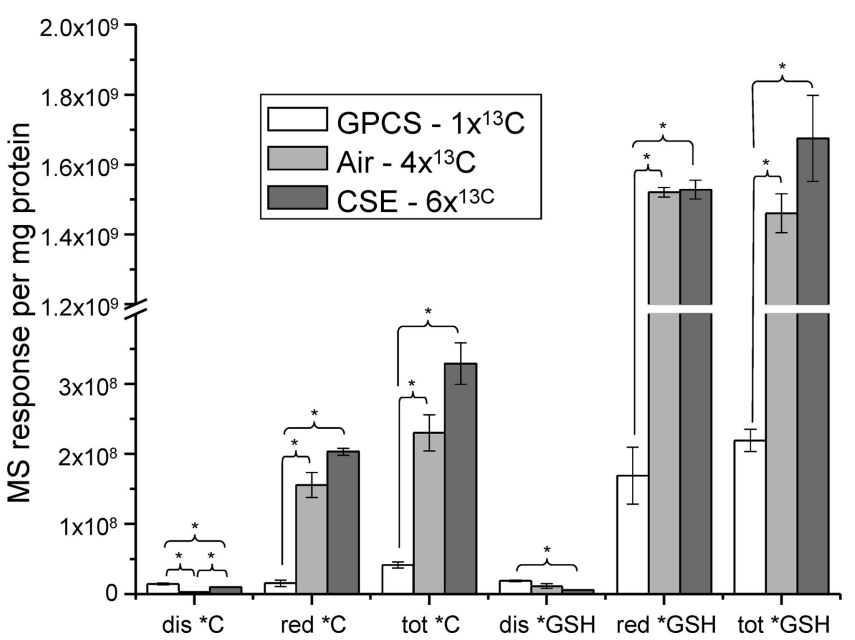

Figure 5. Quantification of thiol-containing species from A549 cell lysates exposed to air, gas-phase cigarette smoke (GPCS), and cigarette smoke extract (CSE) based on stable isotope labeling. MS signals were corrected with respect to protein concentrations. MS responses are expressed per milligram of protein. Averages and standard deviations were established based on 3 biological replicates ( 2 for CSE). Asterisks indicate a significant difference $(p<0.05)$ based on the Kruskal-Wallis test (dis ${ }^{*} \mathrm{C} /$ dis ${ }^{*} \mathrm{GSH}$, cysteine/ glutathione disulfides; red * $\mathrm{C} / \mathrm{red}{ }^{*} \mathrm{GSH}$, cysteine/glutathione reduced as free thiol; tot ${ }^{*} \mathrm{C} /$ tot ${ }^{*} \mathrm{Gsh}$, total cysteine/glutathione (disulfide + free-thiols)).

or that the GPCS treatment was so toxic that some cells had already entered necrosis. It was important to ensure that remainders of the cell culture medium, which contains free amino acids, did not interfere with our measurements. To this end, we analyzed the final wash solution for amino acids and found that there was no interference with the exception of glycine, which was consequently eliminated from quantification.

Since our previous results, ${ }^{24}$ together with other reports, ${ }^{25,26}$ showed that thiol-containing species (notably glutathione) are particularly affected by cigarette smoke, we quantified these species with and without a differential alkylation step to discriminate free thiol forms from disulfides. Thiol-containing compounds are of particular interest when it comes to oxidative stress, since they serve as first-line defense. ${ }^{27-29}$ When samples (not treated first with iodoacetamide) were labeled with PEG-OPFP and incubated in a boiling water bath to remove nonspecific labeling, free thiols were oxidized to form disulfide bonds. This approach is thus not suitable to assess the ratio between free and disulfidebonded thiols. Through reduction with DTT all disulfides were reduced, allowing quantification of the total level of thiol-containing species independently of their initial redox state (total cysteine and total glutathione levels). By first treating the samples with iodoacetamide, only free thiols will be alkylated, whereas the disulfide forms remain unaffected. Following incubation in a

(24) van der Toorn, M.; Smit-de Vries, M. P.; Slebos, D. J.; de Bruin, H. G.; Abello, N.; van Oosterhout, A. J.; Bischoff, R.; Kauffman, H. F. Am. J. Physiol. Lung Cell. Mol. Physiol. 2007, 293, L1156-L1162.

(25) Reddy, S.; Finkelstein, E. I.; Wong, P. S.; Phung, A.; Cross, C. E.; van der Vliet, A. Free Radical Biol. Med. 2002, 33, 1490-1498.

(26) Yalcin, T.; Gabryelski, W.; Li, L. Anal. Chem. 2000, 72, 3847-3852.

(27) Dickinson, D. A.; Forman, H. J. Biochem. Pharmacol. 2002, 64, 1019-1026.

(28) Nolin, T. D.; McMenamin, M. E.; Himmelfarb, J. J. Chromatogr., B: Anal. Technol. Biomed. Life Sci. 2007, 852, 554-561.

(29) Patsoukis, N.; Georgiou, C. D. Anal. Bioanal. Chem. 2004, 378, 17831792.
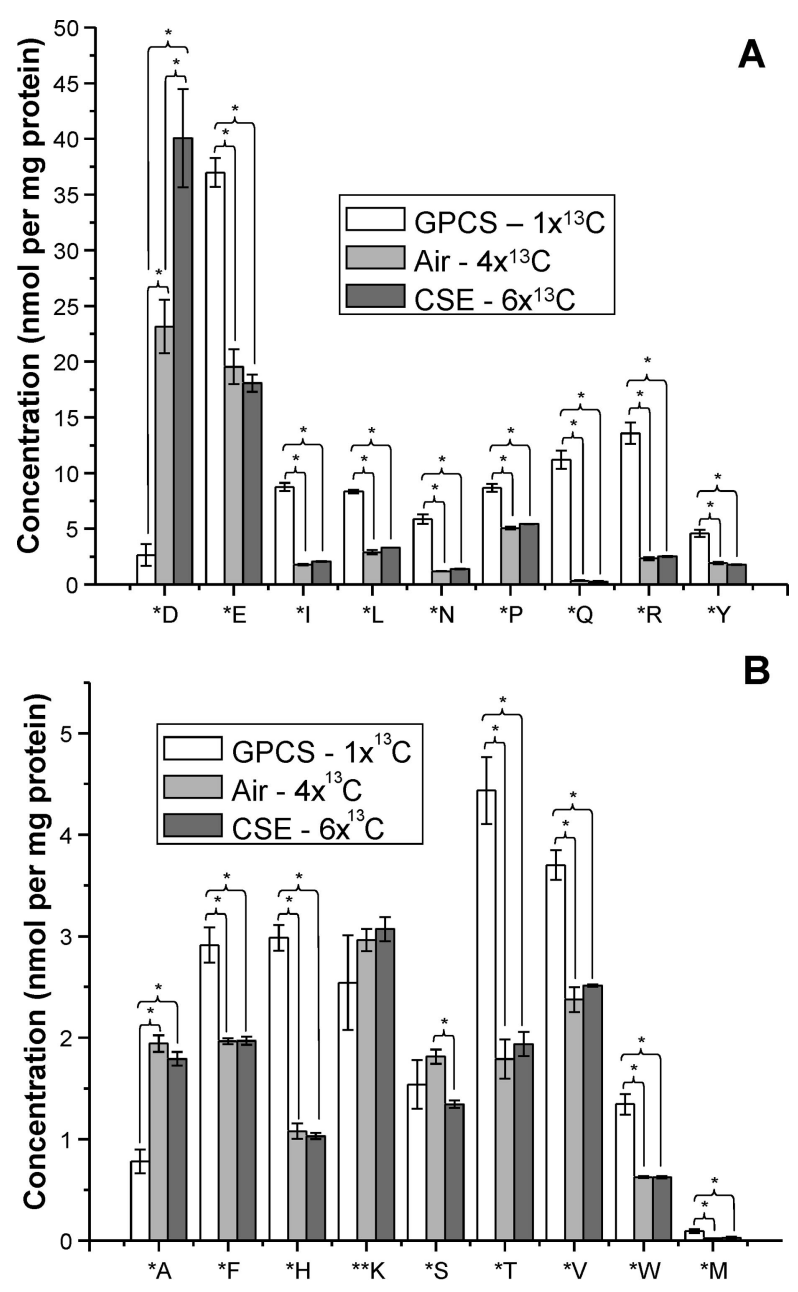

Figure 6. Quantification by stable isotope labeling of amino acids from A549 cell lysates exposed to air, gas-phase cigarette smoke (GPCS) and cigarette smoke extract (CSE). (A) Singly labeled amino acids aspartic acid $\left({ }^{\star} D\right)$, glutamic acid $\left({ }^{*} E\right)$, isoleucine $\left({ }^{*} I\right)$, leucine $\left({ }^{\star} \mathrm{L}\right)$, asparagine $\left({ }^{\star} \mathrm{N}\right)$, proline $\left({ }^{\star} \mathrm{P}\right)$, glutamine $\left({ }^{\star} \mathrm{Q}\right)$, arginine $\left({ }^{\star} \mathrm{R}\right)$, and tyrosine $\left({ }^{\star} Y\right)$. (B) Singly labeled amino acids alanine $\left({ }^{\star} A\right)$, phenylalanine $\left({ }^{*} \mathrm{~F}\right)$, histidine $\left({ }^{*} \mathrm{H}\right)$, serine $\left({ }^{*} \mathrm{~S}\right)$, threonine $\left({ }^{*} \mathrm{~T}\right)$, valine $\left({ }^{*} \mathrm{~V}\right)$, tryptophan $\left({ }^{\star} \mathrm{W}\right)$, and methionine $\left({ }^{\star} \mathrm{M}\right) .{ }^{*} \mathrm{~K}$ stands for doubly labeled lysine. Mass spectrometric signals were converted to molar concentrations using calibration curve equations (see Figures S-2-S-22 in Supporting Information) and subsequently corrected with respect to the total protein concentrations. Results are expressed in $\mathrm{nmol}$ of amino acid/mg of protein. Averages and standard deviations were established based on 3 biological replicates (2 for CSE). Asterisks indicate a significant difference $(p<0.05)$ with the Kruskal-Wallis test.

boiling water bath and subsequent reduction, free thiols will appear in their alkylated forms while thiols that were initially disulfide-bonded will now contain a free, nonalkylated thiol. Both forms can be discriminated by LC-MS and quantified separately. While the intracellular levels of the disulfide forms were rather low irrespective of the treatment (although there were statistical differences), a major drop in the relative amounts of both reduced (free thiol) and total fractions of cysteine and glutathione was observed upon GPCS exposure while this was not the case for CSE (Figure 5). These results are in accordance with earlier findings ${ }^{24,25,30}$ showing that cigarette smoke does not (signifi-

(30) Park, E. M.; Park, Y. M.; Gwak, Y. S. Free Radical Biol. Med. 1998, 25, 79-86. 
cantly) oxidize glutathione to its disulfide form but rather inactivates it by irreversible reactions with reactive aldehydes.

For most of the other amino acids (Figure 6A and B), the content in air- and CSE-treated cells was roughly similar while the content in GPCS-treated cells was strongly increased, from 2to 30 -fold in the case of glutamine. This increase in free amino acids in cells exposed to GPCS has to our knowledge not been reported before, and the reason is presently unclear. Two hypotheses may explain this observation: (a) cells increase their intake of amino acids from the culture medium as a result of exposure to GPCS, and (b) more proteins are ubiquitinated and targeted for degradation by the proteasome to supply free amino acids in conjunction with increased proteasome activity. Indeed most of the amino acids that were observed to be increased upon GPCS-induced oxidative stress are glucogenic, meaning that they can be converted into glucose to serve as energy source for the stressed cells. Interestingly, this effect was only observed in GPCS but not in CSE-treated cells, likely because some reactive species (lipophilic compounds, free radicals) were no longer present in CSE due to the extraction with culture medium. Alternatively, the species responsible for the effect observed with GPCS may have been scavenged by molecules present in the culture medium, such as glutathione or cysteine.

The increase in intracellular amino acid levels upon GPCS exposure is not a general phenomenon. For example, lysine showed no significant change between any of the three conditions while alanine and more so aspartic acid actually decreased in GPCS-treated cells (Figure 6A and B). The fact that aspartic acid was strongly decreased whereas the chemically similar glutamic acid was increased argues against an explanation that is purely based on the chemical reactivity of these amino acids but rather in favor of a biochemical response of the cells. Aspartic acid may be consumed as part of an activated, energy-producing metabolic pathway such as the urea cycle or the malate-aspartate shuttle, on top of being itself a glucogenic amino acid. Further work is needed to elucidate the biological mechanism but this was not the focus of the present work.

\section{CONCLUSION}

PEG-OPFPs constitute a new family of poly(ethylene glycol)based reagents for the stable isotope labeling of primary amine- containing molecules. Hence, important metabolites such as the natural amino acids and glutathione can be monitored with applications going beyond the presented biochemical experiments. Additionally other important biomolecules that usually escape reversed-phase LC-MS analysis, such as oligoamines, spermine, and spermidine, may be quantified. The synthesis of the reagents is based on a sequential buildup of the PEG chain that allows introduction of increasing numbers of ${ }^{13} \mathrm{C}$ atoms offering extensive multiplexing possibilities that go beyond presently available strategies. Derivatization of small hydrophilic molecules such as amino acids resulted in their successful retention on widely used $\mathrm{C}_{18}$ reversed-phase columns without producing retention time shifts between isotopologues. The mass increase moved low molecular weight metabolites out of the often noisy, low $\mathrm{m} / \mathrm{z}$ region of mass spectra. The MS response was enhanced as indicated by the analysis of free versus derivatized tryptophan. Further studies are required to corroborate this observation. The described stable isotope labeling strategy can also be applied to peptides, as shown in ongoing studies focusing on proteomics applications and may be adapted to the labeling of other functional groups.

\section{ACKNOWLEDGMENT}

The authors thank Prof. Dr. Geny M.M. Groothuis (Department of Pharmacokinetics and Drug Delivery, University of Groningen, Groningen, The Netherlands) for the use of the sonicator, and Dr. Peter Horvatovich (Department of Analytical Biochemistry, University of Groningen, Groningen, The Netherlands) for helpful discussions about statistics. N.A. is the recipient of an Ubbo Emmius fellowship (University of Groningen). The Netherlands Proteomics Center (NPC) is gratefully acknowledged for its financial support. N.A. and P.P.G. contributed equally to this work.

\section{SUPPORTING INFORMATION AVAILABLE}

Additional information as noted in text. This material is available free of charge via the Internet at http://pubs.acs.org.

Received for review June 15, 2008. Accepted September 12, 2008.

AC801215C 